\title{
Coherence and incoherence in extended broadband triplet interaction
}

\author{
G. I. de Oliveira \\ Departamento de Física, Centro de Ciências Exatas e Tecnologia Universidade Federal do Mato Grosso do Sul, Postal 549, \\ 79070-900 Campo Grande, MS, Brazil \\ F. B. Rizzato \\ Instituto de Física, Universidade Federal do Rio Grande do Sul, Postal 15051, 91501-970 Porto Alegre, RS, Brazil
}

(Received 19 October 2007; published 29 January 2008)

\begin{abstract}
We study the transition from coherent to incoherent dynamics in a nonlinear triplet of broadband combs of waves. Expanding the analysis of previous works, we investigate what happens when the band of available modes is much larger than that of the initial narrower combs within which the nonlinear interaction is not subjected to selection rules involving wave momenta. Here selection rules are present and active, and we examine how and when coherence can be defined.
\end{abstract}

DOI: 10.1103/PhysRevE.77.016607

PACS number(s): 42.25.Kb, 05.45.- a, 52.35. $-\mathrm{g}$

\section{INTRODUCTION}

Wave-triplet interactions model a vast number of cases where the nonlinear wave dynamics of physical systems can be described in terms of three dominant modes. The interaction is seen in a variety of situations, ranging from threewave interactions in laser-plasma and optical systems to pulsar emission of electromagnetic radiation, including wave interactions in fluids and in several other settings [1-4].

The conservative interaction, which will be our focus here, is more easily handled when the interaction involves only the three pure modes of the triplet. However, a more realistic view should allow for a microscopic description, where each of the pure modes is replaced with a comb with many submodes. This has been done in a number of papers [5-8] where several results have been derived along recent years. The main lesson one learns is that the dynamics can be correctly described in terms of three single or central modes, as long as nonlinearities are strong enough to lock all submodes into a single coherent mode. For practical purposes the coherent modes can then be viewed as pure modes of the triplet interaction. On the other hand, if locking is not effective, each of the submodes follows its own linear dynamics and coherence is lost. Random phase approximations can then be invoked to analyze the problem [9], but the concept of a pure triplet has to be abandoned.

A recent paper [8] shows how combs of modes can be very naturally formed in a wave system: the essential requirement, as we shall review, is that the nonlinear interaction take place under spatially inhomogeneous conditions. When the inhomogeneity is present, wave vector matching among the interacting modes need not to be exact since it includes the reciprocal vectors of the inhomogeneities. What happens then is that even if the initial conditions involve a small number of wave modes, in a very short time interval the initial modes scatter off the inhomogeneities, creating groups of many modes, the initial combs. Another but equivalent way to see how combs are related to inhomogeneities is to realize that in the interaction of tightly packed group of modes, neighboring wave vectors cannot be properly resolved in finite-size spatial scales, a typical occurrence in experimental settings $[10,11]$. In this case whole groups of modes with similar wave vectors are altogether excited, forming the combs. The interaction acquires the aspect of a mean-field theory, where modes of one comb interact with averages taken over modes of the remaining combs [10].

In the past, models for wave combs were based on combs with a fixed number of modes. Once the combs were formed, submodes could evolve in time, but always preserving a prefixed total number within each of the combs. A recent analysis [12] shows that combs with a prefixed number of modes cannot actually maintain this number if the interaction takes place in a homogeneous environment. As one may conclude from the comments above, this happens by virtue of the fact that homogeneity is unable to create a natural wave vector scale which could accommodate a given finite number of modes. Reference [12] indeed shows that as the wave dynamics develops, more and more modes are gradually excited and included in the interaction.

This leads us to the central question of the present analysis: namely, can the wave interaction in inhomogeneous settings be well described with combs of a finite number of modes? We shall see that the answer depends on the time scales and the wave vector scales one is interested in.

The plan of the paper is the following. In Sec. II we first define a convenient interaction model, allowing for an inhomogeneous environment, and explore how the model can be used to create the picture of interacting combs with fixed number of modes, simultaneously analyzing its inherent limitations. In Sec. III we examine what happens when the constraint of a constant prefixed number of modes is relaxed. In Sec. IV we summarize our results.

\section{MODEL}

The investigation starts as we consider the set of fully dimensionless space-time equations governing the decay of mode 1 into modes 2 and 3:

$$
\begin{gathered}
i \partial_{t} a_{1}(x, t)+i v_{g 1} \partial_{x} a_{1}(x, t)=s(x) a_{2}(x, t) a_{3}(x, t), \\
i \partial_{t} a_{2}(x, t)+i v_{g 2} \partial_{x} a_{2}(x, t)=s(x) a_{1}(x, t) a_{3}(x, t)^{*},
\end{gathered}
$$




$$
i \partial_{t} a_{3}(x, t)+i v_{g 3} \partial_{x} a_{3}(x, t)=s(x) a_{1}(x, t) a_{2}(x, t)^{*} .
$$

Set (1)-(3) actually describes the slow modulational dynamics for the complex wave amplitudes $a_{p}(x, t)(p=1,2,3)$ of corresponding carrier waves whose frequencies and wave vectors are matched. The combs are thus the multitude of sideband modes forming around each of three highfrequency carriers. $i^{2}=-1$, and the real function $s(x)$ is the spatially dependent form factor introducing inhomogeneity into the problem. The function $s(x)$ could be typically associated with inhomogeneous density distributions in plasma systems, for instance.

Let us first of all see how the classical picture of combs with a given number of modes can arise from the basic set. We first need a structure for the function $s(x)$. We define it as an even function centered at $x=0$ and a characteristic half width $l_{s}$, as follows:

$$
\begin{gathered}
s=s\left(x / l_{s}\right)=s\left(|x| / l_{s}\right), \\
s\left(|x| / l_{s} \gg 1\right) \rightarrow 0, \quad s(0)=1,
\end{gathered}
$$

where for mathematical convenience, and with no loss of generality, we assumed a scaling that renders $s(0)=1$. This kind of function restricts the effective interaction region as commented in the Introduction and can be used to introduce the basic wave vector associated with the inhomogeneities of the system in the form $k_{s} \sim 1 / l_{s}$. Now we write each of the waves $a_{p}(x, t)$ as combs of many modes:

$$
a_{p}(x, t)=\int \hat{a}_{p}\left(\kappa_{p}, t\right) e^{i \kappa_{p} x} d \kappa_{p},
$$

where $\kappa_{p}$ denotes the wave vectors of submodes within each comb.

Spatial Fourier analysis of set (1)-(3) produces the following group of equations for the various submodes:

$$
\begin{aligned}
i \dot{\hat{a}}_{1}\left(\kappa_{1}\right)= & v_{g 1} \kappa_{1} \hat{a}_{1}\left(\kappa_{1}\right)+\int_{\kappa_{2}, \kappa_{3}} \hat{s}\left(\kappa_{1}-\kappa_{2}-\kappa_{3}\right) \\
& \times \hat{a}_{2}\left(\kappa_{2}\right) \hat{a}_{3}\left(\kappa_{3}\right) d \kappa_{2} d \kappa_{3}, \\
i \dot{\hat{a}}_{p}\left(\kappa_{2}\right)= & v_{g 2} \kappa_{2} \hat{a}_{2}\left(\kappa_{2}\right)+\int_{\kappa_{1}, \kappa_{3}} \hat{s}\left(\kappa_{1}-\kappa_{2}-\kappa_{3}\right) \\
& \times \hat{a}_{1}\left(\kappa_{1}\right) \hat{a}_{3}\left(\kappa_{3}\right)^{*} d \kappa_{1} d \kappa_{3}, \\
i \dot{\hat{a}}_{3}\left(\kappa_{3}\right)= & v_{g 3} \kappa_{3} \hat{a}_{3}\left(\kappa_{3}\right)+\int_{\kappa_{1}, \kappa_{2}} \hat{s}\left(\kappa_{1}-\kappa_{2}-\kappa_{3}\right) \\
& \times \hat{a}_{1}\left(\kappa_{1}\right) \hat{a}_{2}\left(\kappa_{2}\right) * d \kappa_{1} d \kappa_{2},
\end{aligned}
$$

with

$$
s\left(x / l_{s}\right)=\int_{-\infty}^{\infty} \hat{s}\left(\kappa_{s}\right) e^{i \kappa_{s} x} d \kappa_{s}, \quad \hat{s}\left(\kappa_{s}\right)=\frac{1}{2 \pi} \int_{-\infty}^{\infty} s\left(x / l_{s}\right) e^{-i \kappa_{s} x} d x,
$$

$\hat{s}\left(\kappa_{s}\right)$ also even. One thus sees from the second equation of Eqs. (9) that, in general, wave vector mismatches of magni- tudes up to $\left|\kappa_{1}-\kappa_{2}-\kappa_{3}\right|_{\max } \approx \pi / l_{s}$ among the interacting submodes are allowed. If one defines a bandwidth $\Delta$ in the form $-\Delta / 2<\kappa_{p}<\Delta / 2$, one concludes that all modes initially placed within the bands will interact simultaneously, with no constraints due to selection rules, provided $\pi / l_{s} \sim 3 \Delta / 2$. We shall refer to this regime as the regime of democratic interaction because selection rules are not operative here; under this regime, any three modes within the bands are coupled with the same strength. If $l_{s} \rightarrow \infty$, one recovers the matched selection rule $\kappa_{1}=\kappa_{2}+\kappa_{3}$, but for finite $l_{s}$ 's any triple modes within the bands are connected. The approximate dynamics of bands can be obtained if one assumes $\hat{s}\left(\kappa_{1}-\kappa_{2}-\kappa_{3}\right)$ $\sim \hat{s}(0)$ for $\left|\kappa_{p}\right| \leqslant \Delta / 2$, discarding all modes outside the combs; we note that under this approximation, and considering the normalization choice $s(0)=1$, the first equation of Eqs. (9) informs us that $\hat{s}(0) \sim 1 /(3 \Delta)$. In this case, and moving into the discrete version of our continuum equations with $\kappa_{p=1,2,3} \rightarrow \kappa_{m}=2 \pi m / L \equiv m \kappa_{L}$ ( $m$ is an integer denoting the modal number), $d \kappa_{p} \hat{a}_{p}\left(\kappa_{p}\right)=\kappa_{L} \hat{a}_{p}\left(\kappa_{p}\right)=(2 \pi / L) \hat{a}_{p}\left(\kappa_{p}\right) \rightarrow \hat{a}_{p m}$, and $L$ as the system length, one arrives at the set already explored by various authors $[5-7,10,13]$ :

$$
\begin{gathered}
i \dot{\hat{a}}_{1 q}=v_{g 1} \kappa_{q} \hat{a}_{1 q}+\frac{1}{3 N_{\Delta m, n}} \sum_{2 m} \hat{a}_{2 m} \hat{a}_{3 n}, \\
i \dot{\hat{a}}_{2 m}=v_{g 2} \kappa_{m} \hat{a}_{2 m}+\frac{1}{3 N_{\Delta q, n}} \sum_{1 q} \hat{a}_{3 n}^{*}, \\
i \dot{\hat{a}}_{3 n}=v_{g 3} \kappa_{n} \hat{a}_{3 n}+\frac{1}{3 N_{\Delta q, m}} \sum_{1 q} \hat{a}_{1 q} \hat{a}_{2 m}^{*} .
\end{gathered}
$$

To obtain set (10)-(12) the prefactor $\kappa_{L} /(3 \Delta)=(1 / 3)\left(\kappa_{L} / \Delta\right)$ of the nonlinear terms in the discrete version is written as $(1 / 3) /\left(1 / N_{\Delta}\right), N_{\Delta} \equiv \Delta / \kappa_{L}$ being therefore a measure of the number of modes composing the combs in the Fourier reciprocal space; the factor of 3 can be absorbed into convenient rescalings. As mentioned, set (10)-(12) comprises the classical form of the broadband triplet interaction, where selection rules among the wave vectors are absent in virtue of finite size of the interaction region. Several interesting results have been obtained, the most prominent of which concerning the competition between the linear and nonlinear terms. If the linear bandwidth terms associated with the group velocities are absent, one shows that in steady state the wave systems oscillates with a single nonlinear frequency $\Omega$. If $|\Omega|$ is larger than the largest linear frequency $v_{g} \Delta / 2$ (when unnecessary, modal and comb subindexes are occasionally suppressed to simplify notation), a phase-locking mechanism is present, preventing an initially coherent comb from decohering. In general, when a linear bandwidth is present a time propagator $g(t)$ can be constructed for the total amplitude, or macroscopic field of each comb,

$$
A_{p} \equiv \sum_{j} \hat{a}_{p j}
$$

in the form $[8]$ 


$$
g \rightarrow\left\{\begin{array}{l}
g(t)=\frac{1}{\Delta} \int_{-\Delta / 2}^{\Delta / 2}-i e^{i v_{g} \kappa t} d \kappa=-i \frac{\sin \left(\frac{v_{g} t \Delta}{2}\right)}{\frac{v_{g} t \Delta}{2}} \\
\hat{g}(\omega)=\frac{\ln \left[\left(\omega-v_{g} \Delta / 2\right)^{2}\right]-\ln \left[\left(\omega+v_{g} \Delta / 2\right)^{2}\right]}{2 v_{g} \Delta}-\frac{i \pi \operatorname{sign}\left(v_{g} \Delta / 2-\omega\right)+i \pi \operatorname{sign}\left(v_{g} \Delta / 2+\omega\right)}{2 v_{g} \Delta} \quad \text { (frequency domain). }
\end{array}\right.
$$

The factor $-i \exp \left(i v_{g} \kappa t\right)$ in the time domain expression is essentially the propagator for the microscopic mode with wave vector $\kappa$, and the total propagator is obtained through an integration over the whole comb. If in the second equation of Eqs. (14) one identifies the Fourier frequency $\omega$ with the dominant nonlinear frequency $\Omega$, the conclusion is that a dissipative-like term arises whenever $|\Omega|<v_{g} \Delta / 2$. In extreme nonlinear cases with $|\Omega|>v_{g} \Delta / 2$, coherence is preserved. In fact, a relatively straightforward procedure involving expansion of $\hat{g}$ around $\Omega$ and a Fourier inversion from frequency to time domain allows us to write a coupled set for the macroscopic fields [8] which gives a good qualitative view of the dynamics in the democratic regime:

$$
\begin{aligned}
& i \dot{A}_{1} \approx \beta_{1} A_{1}+\frac{1}{3} A_{2} A_{3}, \\
& i \dot{A}_{2} \approx \beta_{2} A_{2}+\frac{1}{3} A_{1} A_{3}^{*}, \\
& i \dot{A}_{3} \approx \beta_{3} A_{3}+\frac{1}{3} A_{1} A_{2}^{*},
\end{aligned}
$$

where $\beta \rightarrow\left(v_{g} \Delta\right)^{2} /(12 \Omega)$ if $v_{g} \Delta \ll \Omega$ and $\beta \rightarrow-i v_{g} \Delta$ if $v_{g} \Delta \gg \Omega$. One sees that given the autonomous aspect of set (15)-(17) one predicts decay (shrinking of volumes in the corresponding phase space) if $\Delta$ becomes larger than the nonlinear frequency.

We shall obtain $\Omega$ explicitly for some cases, but let us first dwell on the role of the width $\Delta$. It is a fixed quantity which corresponds to one-third of the total interaction range defined by the form factor $\hat{s}\left(\kappa_{s}\right)$. The width $\Delta$ contains a number $N_{\Delta}=\Delta / \kappa_{L}$ of modes which in the past were supposed to be the only active modes of the wave system. However, the traditional model set (10)-(12) is only an approximation to the full nonlinear system (6)-(8), where one deliberately discards all modes outside the comb of the given width $\Delta$. The assumption looks right because, as mentioned, modes within the comb are expected to be more strongly and more quickly excited than modes outside. However, when one looks at the full set of equations there is always a nonlinear coupling which may eventually interlace and excite all modes, even those not initially placed inside the combs. In a real system with a band extension naturally much larger than the width $\Delta$, the propagator for the entire macroscopic field should be rewritten as in Eq. (14), but with $\Delta$ replaced with $\Delta_{T}$, the latter quantity representing the total bandwidth available to the modes. Thus, even if $|\Omega|>v_{g} \Delta / 2$, one might still have $|\Omega|<v_{g} \Delta_{T} / 2$, a situation where coherence decay might be present. Of course, if one takes $\Delta_{T}$ as the full bandwidth, and $\Delta_{T}>\Delta$, not all modes will interact democratically and selection rules shall reappear. In that case, previous results must be reevaluated. In particular, from the standpoint of macroscopic modes, the systems ceases to be autonomous since the nonlinear terms can no longer be written only in terms of $A_{1}$, $A_{2}$, and $A_{3}$. Therefore one cannot prove or disprove that volumes in the phase space of the macroscopic modes are shrinking, as happens with the approximate form given by Eqs. (15)-(17). Nevertheless, a dissipative term is present and the macroscopic modes are likely to decay in time-this is what really happens as we show next.

\section{MORE ACCURATE VIEW: THE EXTENDED BROADBAND INTERACTION}

As said, the full set (6)-(8) is equivalent to the its counterpart spatial set (1)-(3). The connection is relevant because if one discards space derivatives, exact solutions can be obtained. These exact solutions form the basis for further progress as one includes the space derivatives.

\section{A. Neglecting space derivatives}

Taking $v_{g p} \partial_{x} \rightarrow 0$ in the Eqs. (1)-(3) a stationary solution can be obtained in the form $A_{p}(x, t)=\rho_{p}(x) \exp \left[i \phi_{p}(x, t)\right]$, where $\quad \phi_{1}=-2 \rho_{1}(x) s(x) t, \quad \phi_{2,3}=-\rho_{1}(x) s(x) t, \quad$ and $\quad \rho_{2,3}$ $=\sqrt{2} \rho_{1}(x)$ and where $\rho_{1}(x)$ is an arbitrary $x$-dependent function; we note that in the stationary state phases depend on time, but amplitudes do not. Once $\rho_{1}(x)$ is defined, the complete solution is automatically found. And once the spacetime solution is found, Fourier transforms can be used to move into the reciprocal space. To further specify the system with localized initial conditions, both in the real and reciprocal spaces, we shall make the following choice for the combs and the form factor $s$ in the spatial representation:

$$
\begin{gathered}
\rho_{1}(x)=\rho_{0} \exp \left(-x^{2} / l_{\rho}^{2}\right), \\
s\left(x / l_{s}\right)=\exp \left(-x^{2} / l_{s}^{2}\right) .
\end{gathered}
$$

The comb thus defined has width $\sim 1 / l_{\rho}$ in the reciprocal space, and in order that its modes interact democratically at least initially, we require $1 / l_{s} \geqslant 3 / l_{\rho}$ as explained earlier-in 
all numerical work we actually take $1 / l_{s}=3 / l_{\rho}$. We shall also assume that $\Delta_{T} \gg \Delta$ and write for the exponential distribution $\Delta=2 \pi / l_{\rho}$.

Independently of the choices we make for $\rho_{1}(x)$ and $s(x)$ we are already in position to define coherence in the present case. We simply note that since

$$
a_{p}(x, t)=\sum_{j} \hat{a}_{p j} e^{i \kappa_{j} x},
$$

it is true that the macroscopic fields $A_{p}$ introduced earlier in Eq. (13) obey simple expressions-we write down the one obeyed by $A_{1}$ :

$$
A_{1}=\sum_{j} \hat{a}_{1 j}=\rho_{0} \exp \left(-2 i \rho_{0} t\right) .
$$

From the equation above we see that the macroscopic field oscillates harmonically with frequency $\Omega \equiv-2 \rho_{0}$ and with constant amplitude $\rho_{0}$. This is what we shall take as a coherent state: a nondecaying macroscopic mode oscillating with constant amplitude and constant frequency. The question that poses itself here is to determine how many microscopic modes actually participate in the coherent state. In other words, would it be true to assume that only the modes inside the initially defined combs are active? At a first glance one might suspect the answer would be positive since those are the modes interacting more strongly in the system. However, we had already pointed out that due to the nonlinear cascading structure of the interaction, some energy may flow from low to high wave vectors, and this is what actually happens. This can be seen more formally with the help of some tools. Keeping focus on the first comb, one first chooses a range $D$ defined by $-D / 2<\kappa<D / 2$ and performs a partial summation over its internal modes,

$$
\begin{aligned}
\mathcal{I}_{D} & \equiv \int_{-D / 2}^{D / 2} a_{1}\left(\kappa_{1}\right) d \kappa_{1} \\
& =\frac{1}{2 \pi} \int_{-D / 2}^{D / 2} \int_{-\infty}^{\infty} e^{-i \kappa_{1} x} \rho_{1}(x) \exp \left[-2 i \rho_{1}(x) s(x) t\right] d x d \kappa_{1} .
\end{aligned}
$$

For a finite band $D$, the integral over $\kappa_{1}$, performed first, yields a $\delta$-like structure as a function of $x$, with height $D / 2 \pi$ and width $2 \pi / D$. If one supposes $1 / D$ small, the remaining integration over $x$ can be done with the help of a saddle approximation near $x=0$ where the space derivative of fields and form factor vanish. The final result can be written in the form

$$
\mathcal{I}_{D} \sim e^{-2 i \rho_{0} t} \sqrt{\rho_{0}} D \frac{\operatorname{Erfi}\left[e^{3 i \pi / 4} \pi \sqrt{2 \rho_{0} t s^{\prime \prime}(0) / 2} / D\right]}{\sqrt{t s^{\prime \prime}(0)}},
$$

where $s^{\prime \prime} \equiv d^{2} s / d x^{2}$, where $\operatorname{Erfi}(\chi)$ denotes the imaginary error function as a function of argument $\chi$ and where we recall that $s(x)$ varies faster than $\rho_{1}(x)$. We see that all depends on the behavior of the imaginary error function for large and small arguments. If $|\chi| \ll 1, \operatorname{Erfi}(\chi) \sim \chi$, and if $|\chi| \gg 1$, $\operatorname{Erfi}(\chi) \sim i$. One therefore concludes that

$$
\left|\mathcal{I}_{D}\right| \sim \begin{cases}\text { constant } & \text { when } t \lesssim l_{s}^{2} D^{2} / 2 \pi^{2} \rho_{0}, \\ \frac{1}{\sqrt{t}} & \text { when } t>l_{s}^{2} D^{2} / 2 \pi^{2} \rho_{0} .\end{cases}
$$

In other words, given a range $D$ there is an intrinsic limiting time for coherence,

$$
\tau_{D} \equiv \frac{D^{2} l_{s}^{2}}{2 \pi^{2} \rho_{0}},
$$

where by intrinsic we understand the limiting time obtained in the absence of the linear-frequency bandwidth-i.e., by taking $v_{g}=0$. We know from our discussion regarding Eq. (9) that $l_{s} \sim \pi /(3 \Delta / 2)$, so the intrinsic coherence time for modes within the original packet would be given by $\tau_{D=\Delta} \sim 1 / \rho_{0}$ which is relatively small since this is essentially the period of the nonlinear wave. Our conclusion is that the initial packet can be hardly called a coherent structure even in the absence of the frequency bandwidth. The collection of modes that could be seen as a coherent structure is any one where $D \gg \Delta$. In that case it is still true that decay will be present, but for all practical purposes $\tau_{D}$ would be so much larger than the period of the nonlinear wave that a physical setting or equipment resolving modes up to $\kappa \sim D$ would perceive the wave system as coherent.

A second important time scale has to be defined for the wave system. It is the time scale of the excitation of individual modes in reciprocal space. Looking again at the first comb - the reasonings are similar for the other two-we first recall the expression $A_{1}(x, t)=\rho_{1}(x) \exp \left[i \phi_{1}(x, t)\right]$ for the steady-state field. $\rho_{1}(x)$ is constant in time, and the phase $\phi_{1}(x)=-2 \rho_{1}(x) s(x) t$ depends both on the spatial coordinate and time. If one evaluates the phase gradient $\partial \phi_{1} / \partial x$ and looks at the maximum of this quantity as the largest wave vector involved in the dynamics, one derives the relation

$$
\left|\kappa_{\max }\right| \sim\left(2 \rho_{0} / l_{s}\right) t,
$$

which shows that the packet spreads over reciprocal space at a rate $\pm 2 \rho_{0} / l_{s}$. The time for excitation of any particular wave vector $\kappa_{\max }$ is thus $\tau_{\text {exc }}\left(\kappa_{\max }\right)=\kappa_{\max } l_{s} / 2 \rho_{0}$. If we take $\kappa_{\max }=D / 2$, we see that for the typical case $D l_{s} \gg 1$, $\tau_{D} \gg \tau_{e x c}\left(\kappa_{\max }=D / 2\right)$, which means that in the absence of linear-frequency bandwidths, the coherence time of a packet of range $D$ is in general much longer than the time required to activate the modes at the borders of the packet.

In Fig. 1 we display the contrasting behaviors for $\mathcal{I}_{D}$ in the cases $D l_{s} \sim 1$ and $D l_{s} \gg 1$. In the simulations we integrate set (1)-(3) with a pseudospectral method, using a grid of length $L=N=2^{15}, N$ denoting the number of nodes which for scaling simplicity is equal to the length. In all numerical analyses we use $\rho_{0}=1$; the choice is not restrictive because field scales can always be absorbed in space and time. Considering $l_{s}=2^{9}$ and $l_{\rho}=3 l_{s}$, panel (a) displays the case $D=2 \pi / L \times 2^{8}$, for which $D l_{s}=2 \pi \times 2^{2}$ and $\tau_{D} \sim 32$, while in panel (b), $D l_{s}=2 \pi \times 2^{6}$, for which $\tau_{D} \sim 8200$. Panel (a) reveals a fast decay, but coherence is far more persistent in panel (b). It is noticeable that in panel (b) the function $\mathcal{I}_{D}$, although initially laminar, develops slight modulations after a very sharp instant along the time axis. This very sharp 


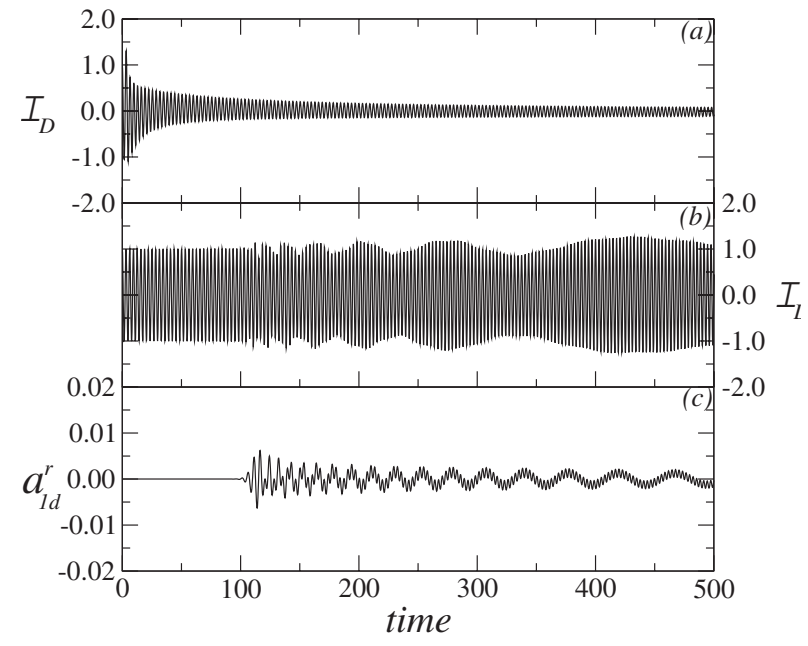

FIG. 1. $\mathcal{I}_{D}$ as a function of time for $D l_{s}=2 \pi \times 2^{2}$ in (a) and for $D l_{s}=2 \pi \times 2^{6}$ in (b). In (c) we show the times series for the real part of the borderline mode with wave vector $\kappa=D / 2, l_{s}=2^{9}$, and $l_{\rho}=3 l_{s}$. The group velocity is zero for all waves, and all quantities are dimensionless.

instant corresponds to $t=\tau_{\text {exc }}(D / 2)$. Indeed, the excitation time reads $\tau_{\text {exc }}(D / 2)=100.5$ in this particular instance. This is confirmed in panel (c) where, for the same parameters as panel (b), we show the time evolution of the real part of mode with wave vector $D / 2$. The superscript $r$ means "real part" and the submodal index $d$ reads $d \equiv(D / 2) /(2 \pi / L)$ $=2^{11}$ in this case, as defined in the context of the discrete equations (10)-(12). We emphasize that as mode $\kappa=D / 2$ is excited, the coherence of the packet $D$, although undergoing a modulational process, does not decay.

Of course, the presence of a bandwidth for the linear frequencies may change the entire picture, and this is the subject of the next section.

\section{B. Effects of space derivatives and the associated linear- frequency bandwidth}

Since the full nonlinear system is not autonomous from the perspective of macroscopic modes, one cannot make very formal predictions about coherence decay due to the frequency bandwidths, like we did in the approximations leading to Eqs. (15)-(17). However, some estimates can still be made.

Let us consider our expression (25) for the maximal wave vector involved in the dynamics. When $\kappa_{\max }$ reaches the value $\kappa_{r}$ corresponding to the resonant frequency, $v_{g 1} \kappa_{r}$ $\equiv 2 \rho_{0}$, coherence is expected to be lost, but now due to resonant effects. Under this circumstance, the largest excited linear frequency would become comparable to the nonlinear triplet frequency $\Omega$ and coherent nonlinear effects would be no longer dominant. The time to attain resonance, let us call it $\tau_{r} \equiv \tau_{\text {exc }}\left(\kappa_{r}\right)$, can be obtained as one uses $\kappa_{\max }=\kappa_{r}$ in Eq. (25):

$$
\tau_{r} \equiv l_{s} / v_{g 1} \cdot
$$

A given collection of modes of range $D$ will remain coherent as long as $t<\tau_{c o h} \equiv \min \left\{\tau_{D}, \tau_{r}\right\}$. To illustrate this point, let us

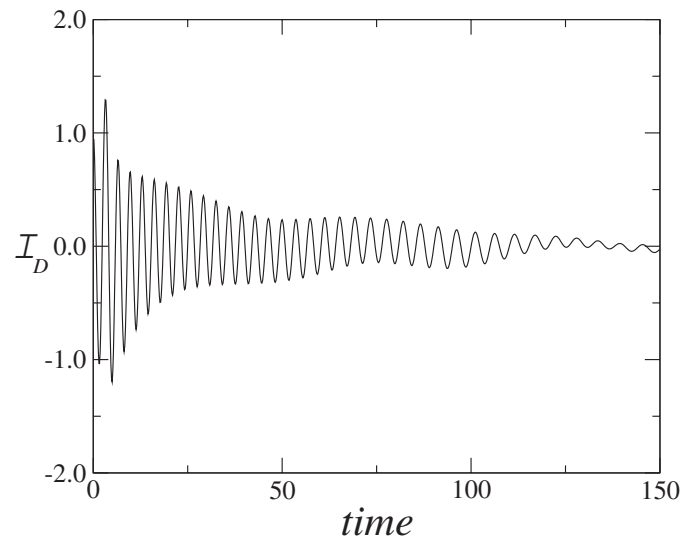

FIG. 2. Coherence decay due to the resonant effect. Parameters are those of panel (b) of Fig. 1, with the exception of $v_{g 1}$ which here reads $v_{g 1}=1 /\left(2^{9} \kappa_{L}\right)$, defining a resonant time $\tau_{r} \approx 50$.

take the case analyzed in the panel (b) of Fig. 1. In that case $\tau_{D}$ is large and we do not expect to see coherence decay soon if the linear frequencies are absent. But now let us add a frequency bandwidth with $v_{g 1}$ chosen such that a given mode of the spectrum becomes resonant with $\tau_{r}<\tau_{D}$; we achieve this requirement with $v_{g 1}=1 /\left(\kappa_{L} 2^{9}\right)$ which yields $\tau_{r} \approx 50<\tau_{D}$. For completeness we take $v_{g 1,2}=0$ which corresponds to one wave moving relatively to the other two. The setting would be of relevance to Brillouin scattering, for instance, where two electromagnetic waves with the same group velocity interact with a slower ion wave; we would be examining the process in the frame where the electromagnetic wave is stationary. The resulting dynamics is then displayed in Fig. 2, where one clearly sees a fast decay whereas for $v_{g}=0$ one sees persistent coherence as previously shown in Fig. 1(b).

Expressions (24) and (26) therefore provide us with a simple tool to make estimates on the circumstances allowing coherence to be seen in the nonlinear triplet system. Once one has defined an extended comb distributed over a range $-D / 2<\kappa<D / 2$ of wave vectors with $D / \Delta \gg 1$ and once one knows the group velocity $v_{g}$ for this particular class of wave, the coherence time can be obtained.

\section{FINAL REMARKS}

In this paper we developed a technique to investigate coherence in nonlinear triplets, when the available band of modes is much larger than that of the initial combs. If modes remain restricted to their initial combs, the series of approximations outlined in Sec. II allows us to describe the system as an interaction of macroscopic modes. In the presently studied case, one cannot resort to these approximations because initially low-amplitude, idle modes outside the initial range will be gradually excited at a rate $\sim 2 \rho_{0} / l_{s}$, whenever the whole available band is larger than $\Delta$. Coherence in this, perhaps, more realistic case is a little more involved subject to define. One first defines the range $D$ of interest. The range has an intrinsic coherence time $\tau_{D}$ defined in the absence of any frequency bandwidth-i.e., for $v_{g}=0: \tau_{D}=D^{2} l_{s}^{2} /\left(2 \pi^{2} \rho_{0}\right)$. $\tau_{D}$ is the largest coherence time of a collection of modes 
contained within the limits $-D / 2<\kappa<D / 2$. Then, once the range $D$ is defined, one has to look at the excitation time $\tau_{r}$ of the resonant mode, which does not necessarily belong to the range $D$; we found that $\tau_{r}=l_{s} / v_{g}$. Gathering together both time scales, the final conclusion is that the coherence time $\tau_{\text {coh }}$ satisfies $\tau_{\text {coh }}=\min \left\{\tau_{D}, \tau_{r}\right\}$. We have also observed and stressed that coherence gains some substantial meaning only when several nonlinear oscillations occur prior to $\tau_{D}$. Since in our normalized variables the period of the nonlinear oscillation is $\sim 1 / \rho_{0}$, one concludes that the dynamics resembles a nonlinear phase-locking process only when $D \gg \Delta$ and $v_{g} \Delta \ll \rho_{0}$.

Let us connect our results with those of previous works. Our macroscopic model does not look into fine microscopic scales of size, say, $l_{\text {mic }}$, where discrete effects become relevant. Therefore an upper limit $D_{\max } \sim 1 / l_{\text {mic }}$ does exist be- yond which mode dynamics is naturally attenuated by microscopic effects. One can, however, imagine that modes with wave vectors $|\kappa|>D_{\max } / 2$ are initially small and heavily damped; if this is true, they will be minimally excited during the dynamics. Under these circumstances the condition on $\tau_{D}$ for an inaccessible $D>D_{\max }$ ceases to exist (since $\tau_{D} \rightarrow \infty$ in this case) and we are left only with the condition on the group velocity and linear bandwidth, which is similar to what is discussed in previous investigations. For $D<D_{\max } \tau_{D}$ is finite and physically relevant.

\section{ACKNOWLEDGMENTS}

We acknowledge support by $\mathrm{CNPq}$, Brasil, and by the AFOSR, under Grant No. FA9550-06-1-0345. We also thank illuminating discussions with R. Pakter and A. Serbeto.
[1] P. K. Shukla, N. N. Rao, M. Y. Yu, and N. L. Tsintsadze, Phys. Rep. 138, 1 (1986).

[2] Y. S. Kivshar and B. Malomed, Rev. Mod. Phys. 61, 763 (1989).

[3] A. C.-L. Chian and M. V. Alves, Astrophys. J. Lett. 330, L77 (1988).

[4] F. T. Gratton, G. Gnavi, R. M. O. Galvão, and L. Gomberoff, Phys. Rev. E 55, 3381 (1997).

[5] A. M. Martins and J. T. Mendonça, Phys. Rev. A 31, 3898 (1985).

[6] A. M. Martins and J. T. Mendonça, Phys. Fluids 31, 3286 (1988).

[7] P. M. Drysdale and P. A. Robinson, Phys. Plasmas 9, 4896 (2002); P. A. Robinson and P. M. Drysdale, Phys. Rev. Lett.
77, 2698 (1996).

[8] M. Frichembruder, R. Pakter, and F. B. Rizzato, Physica D 215, 99 (2006).

[9] R. Davidson, Methods in Nonlinear Plasma Theory (Academic Press, New York, 1972).

[10] J. C. Weiland and H. Whilhelmsson, Coherent Nonlinear Interaction of Waves in Plasmas (Pergamon, Oxford, 1977).

[11] J. T. Mendonça, A. Serbeto, R. Bingham, and P. K. Shukla, J. Plasma Phys. 71, 119 (2005).

[12] M. Frichembruder, R. Pakter, and F. B. Rizzato, J. Plasma Phys. 71, 11 (2005).

[13] F. B. Rizzato, R. Pakter, and S. R. Lopes, Phys. Rev. E 68, 056601 (2003). 